\title{
Analysis of the ac SQUID with low inductance and low critical current
}

\section{Sørensen, O. H.}

\section{Published in:}

Journal of Applied Physics

Link to article, DOI:

10.1063/1.322461

Publication date:

1976

\section{Document Version}

Publisher's PDF, also known as Version of record

Link back to DTU Orbit

Citation (APA):

Sørensen, O. H. (1976). Analysis of the ac SQUID with low inductance and low critical current. Journal of Applied Physics, 47(11), 5030-5037. https://doi.org/10.1063/1.322461

\section{General rights}

Copyright and moral rights for the publications made accessible in the public portal are retained by the authors and/or other copyright owners and it is a condition of accessing publications that users recognise and abide by the legal requirements associated with these rights.

- Users may download and print one copy of any publication from the public portal for the purpose of private study or research.

- You may not further distribute the material or use it for any profit-making activity or commercial gain

- You may freely distribute the URL identifying the publication in the public portal

If you believe that this document breaches copyright please contact us providing details, and we will remove access to the work immediately and investigate your claim. 


\title{
Analysis of the ac SQUID with low inductance and low critical current
}

\author{
O. H. Soerensen \\ Physics Laboratory I, The Technical University of Denmark, DK- 2800 Lyngby, Denmark \\ (Received 6 October 1975)
}

\begin{abstract}
The properties of the ac SQUID magnetometer has been analyzed. The results are valid in the lowinductance low-critical-current regime, where the $L_{R} I_{0}$ product is below the value at which the relation between the enclosed and externally applied magnetic dc flux becomes reentrant. The effects of the screening currents circulating in the SQUID ring as well as of the SQUID-ring time constant, $\tau=L_{R} / R$, are taken into account. Here, $L_{R}$ is the SQUID-ring inductance, and $R$ is the shunt resistance in the shunted junction model assumed to describe the weak link. It is shown that for finite values of $\omega \tau$, where $\omega / 2 \pi$ is the pump frequency, the magnetometer response is due to both the flux dependence of the dispersion and of the dissipation in the tank circuit, and that, generally, the two mechanisms contribute simultaneously. Furthermore, for appropriately chosen ac bias conditions they may add constructively with the result that the optimal response occurs at a definite and finite value of $\omega \tau$. If $\omega \tau$ is increased beyond this optimal value the weak link behavior is dominated by the Ohmic current channel implying that only if the shunt conductance contains a term depending on the superconducting phase-as the so-called cosine-phi term predicted by tunneling theory-the SQUID will stay sensitive to magnetic fields for large $\omega \tau$. The influence of the cosine-phi term has been included in the analysis, and as expected it is found to play a significant role particularly for large $\omega \tau$. It is, however, also shown that it is nontrivial to extract its magnitude from experiments based on the behavior of the ac SQUID, and that the recently published observations using this technique must be reinterpreted.
\end{abstract}

PACS numbers: 74.50.T, 85.25., 74.35.

\section{INTRODUCTION}

The superconducting ring closed by a Josephson junction or a "weak link" magnetically coupled to a resonant circuit is an ultrasensitive detector of lowfrequency magnetic field variations. ${ }^{1-7}$ In most practical devices the ring inductance, $L_{R}$, has been greater than $\Phi_{0} / 2 \pi I_{0}$, where $\Phi_{0}$ is the flux quantum and $I_{0}$ is the critical current of the weak link. This means that a multivalued relation exists between $\Phi_{\mathrm{dc}}$, the magnetic dc flux enclosed in the ring, and $\Phi_{x}$, the externally applied flux. The operation of the magnetometer-traditionally named the ac SQUD-is based on a measurement of the energy dissipation accompanying the irreversible transitions of the ring between neighboring flux-quantum states as it is driven around a hysteresis loop in the $\Phi_{d c}-v s-\Phi_{x}$ plane. ${ }^{8}$

Now, SQUID rings with very low inductance have been designed, e.g. , the toroidal SQUID ${ }^{7,9}$ and the multihole SQUID, ${ }^{10}$ and hence it has become possible to operate the ac SQUID as a magnetometer also in the low-inductance regime, $2 \pi L_{R} I_{0} / \Phi_{0}<1$. In the low-inductance regime the dc flux enclosed in the ring is a single-valued function of the external flux, and a consequence of this "single valuedness" is that the analysis of the SQUID system may be carried much further than has been possible for the "hysteretic" mode of operation.

The low-inductance limit with vanishing critical current, $2 \pi L_{R} I_{0} / \Phi_{0} \ll 1$, have been analyzed by Hansma ${ }^{11,12}$ and by Rifkin et al. ${ }^{13}$ The effect of the weaklink normal-state resistance as well as of the phasedependent conductivity ${ }^{14}$ - the so-called cosine-phi term-have been taken into account to first order by these authors.

In this paper an analysis of the ac SQUID operated in the low-inductance regime characterized by the relation $2 \pi L_{R} I_{0} / \Phi_{0} \leqslant 1$ will be presented. In order for the results to be valid for any value of the parameter, $\gamma$ $=2 \pi L_{R} I_{0} / \Phi_{0}$, below the upper limit defined above, it is important that the screening current circulating in the SQUID ring is taken into account. The SQUID ring is coupled magnetically to a resonant circuit driven by an ac source at frequency $\omega / 2 \pi$. The weak link will be described by the resistively shunted junction model of Stewart and McCumber. ${ }^{15}$ Within this model the influence of the intrinsic time constant, $\tau=G L_{R}$, of the SQUID ring will be considered; here, $G$ is the weaklink shunt conductance. Also the phase-dependent conductivity will be included in the model by means of an additional shunt conductance, $\epsilon G \cos \phi$, where $\phi$ is the phase difference across the weak link and $\epsilon$ is an amplitude factor. It is particularly important to learn how the shunt conductance $G$ or equivalently, the associated finite time constant $\tau$ affects the SQUD response, since the sensitivity of the device as a magnetometer increases with increasing pump frequency. This extrapolation can, however, only be valid up to a certain cutoff frequency, which has not previously been taken into account in a quantitative fashion. At high pump frequencies, $\omega \tau>1$, we find that the Ohmic channels actually dominate the weak-link behavior, implying that in this limit the phase-dependent conductivity becomes significant.

In Sec. II an expression for the voltage across the resonant circuit will be derived as a function of the pump frequency, of the amplitude of the ac bias current, and of the externally applied magnetic dc flux. The expression will be valid for any value of the normalized screening flux, $\gamma=2 \pi L_{R} I_{0} / \phi_{0} \leqslant 1$, and the amplitude $\epsilon$ of the cosine-phi conductance relative to the constant shunt conductance may take any value $|\epsilon| \leqslant 1$. 


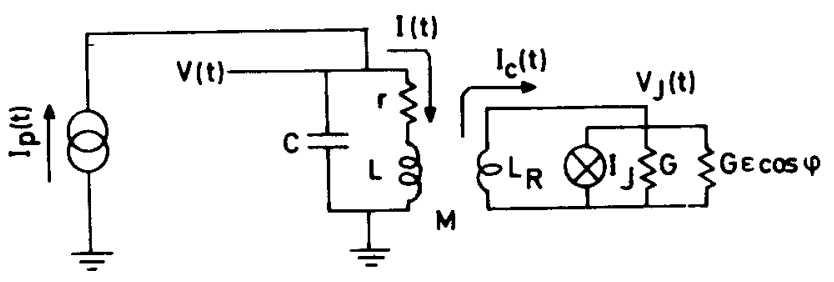

F IG. 1. The magnetometer model circuit. The ac-SQUID is represented by the ring inductance $L_{R}$ and the parallel combination of an ideal Josephson element $I_{J}=I_{0} \sin \phi$, a phase-dependent shunt conductance $\epsilon G \cos \phi$, and a constant shunt conductance $G$.

In Sec. III the dependence of the SQUID response on the pump frequency, and the other external parameters will be evaluated, and it will be shown that an appreciable sensitivity to changes in the magnetic de flux persists to high pump frequencies provided that the cosine-phi amplitude is nonzero. Also, it will be shown that the optimum performance of the magnetometer occurs at a finite value of $\omega \tau$, which depends on $\epsilon$.

In Sec. IV the potential for extracting information on the magnitude of the cosine-phi term from experiments will be discussed. And finally, Sec. V contains a summary and the conclusion.

\section{THEORY}

The model circuit is shown in Fig. 1. The externally controlled parameters are the applied magnetic dc flux and the amplitude and frequency of the ac bias current, $I_{p}(t)$, flowing into the parallel resonant circuit-or tank circuit-with capacitance, $C$, inductance, $L$, and loss resistance, $r$. The SQUID ring with inductance $L_{R}$ is coupled magnetically to the tank coil via the mutual inductance $M$, and the weak link is represented by a parallel combination of an ideal Josephson element $I_{J}$ $=I_{0} \sin \phi$, a shunt conductance $G$, and a phase-dependent conductivity $\epsilon G \cos \phi$.

\section{A. The SOUID ring}

The circulating current, $I_{c}(t)$, induced in the SQUD ring is equal to the current through the weak link and is given by the Josephson equation

$$
I_{c}(t)=I_{0} \sin \phi+G(1+\epsilon \cos \phi) V_{J}(t),
$$

where $I_{0}$ is the junction critical current and $V_{J}(t)$ is the junction voltage. The phase difference $\phi$ is related to the magnetic flux enclosed in the ring, $\Phi_{1 n t}$, by

$$
\phi=2 \pi \Phi_{\text {int }} / \Phi_{0},
$$

and the junction voltage is found from Faraday's induction law

$$
V_{J}=\frac{d}{d t} \Phi_{\text {int }}
$$

which, using Eq. (2), is identical to the Josephson voltage-phase relation.

The external flux $\Phi_{\text {ext }}$ is given by

$$
\Phi_{\text {ext }}=\Phi_{x}-M I(t),
$$

where $I(t)$ is the current flowing through the tank cir- cuit inductance. Finally, the internal flux is related to $\Phi_{\text {ext }}$ by the screening equation

$$
\Phi_{\text {int }}=\Phi_{\text {ext }}-L_{R} I_{c}(t)
$$

When the tank circuit is excited by a current at frequency $\omega / 2 \pi$, the circulating current induced in the SQUID ring will contain a component at that frequency. Due to the nonlinearity of the weak link, higher harmonics and mixing products will be generated, which in turn are fed back into the tank circuit. If we restrict ourselves to work at frequencies close to the resonant frequency of the tank circuit, and if the quality factor of the resonator is moderately high, the frequency component at $\omega / 2 \pi$ is selected. Accordingly, we assume that the current in the inductance $L$ is at a single frequency. With the ac bias current, $I_{p}(t)=I_{p} \sin \omega t$, we seek a solution

$$
I(t)=I \sin (\omega t-\theta),
$$

where the amplitude $I$ and the phase $\theta$ is to be determined.

Using Eqs. (1)-(6) we find that the internal flux is determined by the nonlinear differential equation

$$
\begin{aligned}
& G L_{R}\left(1+\epsilon \cos 2 \pi \Phi_{\mathrm{int}} / \Phi_{0}\right) \dot{\Phi}_{\mathrm{int}}+\Phi_{\mathrm{int}}+L_{R} I_{0} \sin 2 \pi \Phi_{\mathrm{int}} / \Phi_{0} \\
& =\Phi_{x}-M I \sin (\omega t-\theta)
\end{aligned}
$$

where the dot indicates differentiation with respect to time.

In order to keep the analysis on a practicable level we make the basic approximation that only the dc and the fundamental components are important, i. e. ,

$$
\Phi_{\mathrm{int}}=\Phi_{\mathrm{dc}}+\Phi_{\mathrm{ac}} \sin \left(\omega t-\theta^{\prime}\right),
$$

which means that the mixing products between higher harmonics contributing to the amplitude at the fundamental frequency are neglected.

Equation (8) must be inserted into $\mathrm{Eq},(7)$ and the amplitudes, $\Phi_{\mathrm{dc}}$ and $\Phi_{\mathrm{ac}}$, and the phase $\theta^{\prime}$ determined self-consistently. Introducing the normalized flux amplitudes

$$
\begin{array}{ll}
\alpha=-2 \pi \Phi_{\mathrm{ad}} / \Phi_{0}, & \beta=2 \pi \Phi_{\mathrm{d} d} / \Phi_{0}, \\
\gamma=2 \pi L_{R} I_{0} / \Phi_{0}, & \beta_{x}=2 \pi \Phi_{x} / \Phi_{0}
\end{array}
$$

and using the Fourier-Bessel expansions for $\sin \left[\beta-\alpha \sin \left(\omega t-\theta^{\prime}\right)\right]$ and $\cos \left[\beta-\alpha \sin \left(\omega t-\theta^{\prime}\right)\right]$ Eq. (7) becomes

$$
\begin{aligned}
& -\alpha\left[1+\gamma \cos \beta 2 J_{1}(\alpha) / \alpha\right] \sin \left(\omega t-\theta^{\prime}\right) \\
& -\alpha\left\{1+\epsilon \cos \beta\left[J_{0}(\alpha)+J_{2}(\alpha)\right]\right\} \omega \tau \cos \left(\omega t-\theta^{\prime}\right) \\
& +\beta+\gamma J_{0}(\alpha) \sin \beta \\
& \quad=\beta_{x}-2 \pi\left(M I / \Phi_{0}\right) \sin (\omega t-\theta),
\end{aligned}
$$

where $J_{n}(\alpha)$ is the Bessel function of order $n$. Using the identity $J_{0}(\alpha)+J_{2}(\alpha)=2 J_{1}(\alpha) / \alpha$ and separating into time-dependent and time-independent terms we have the two coupled equations

$$
\begin{aligned}
& \alpha\left[1+\gamma 2 J_{1}(\alpha) \cos \beta / \alpha\right] \sin \left(\omega t-\theta^{\prime}\right) \\
& +\alpha\left[1+\epsilon 2 J_{1}(\alpha) \cos \beta / \alpha\right] \omega \tau \cos \left(\omega t-\theta^{\prime}\right) \\
& =2 \pi\left(M I / \Phi_{0}\right) \sin (\omega t-\theta), \\
& \beta+\gamma J_{0}(\alpha) \sin \beta=\beta_{x} .
\end{aligned}
$$


From Eq. $\left(10^{\prime}\right)$ a relation is readily obtained between the amplitude and phase of the ac flux and the amplitude and phase of the tank-coil current. Equation $\left(10^{\prime \prime}\right)$ expresses the external dc flux directly in terms of the dc and ac amplitudes of the internal flux. This equation displays explicitly the depression of the critical current with increasing ac level.

Solving Eq. $\left(10^{\prime}\right)$ for the tank-coil current $I$ in terms of internal normalized ac flux $\alpha$, we obtain

$$
\left(2 \pi M / \Phi_{0}\right) I=\alpha\left\{(1+\gamma \Gamma)^{2}+\left[\omega \tau(1+\epsilon \Gamma)^{2}\right]\right\}^{1 / 2}
$$

and

$$
\theta^{\prime}=\arctan \{\omega \tau(1+\epsilon \Gamma) /(1+\gamma \Gamma)\}+\theta
$$

where

$$
\Gamma=\left[2 J_{1}(\alpha) / \alpha\right] \cos \beta
$$

Originally we defined the low-inductance limit by $\gamma \leqslant 1$. As a consequence of Eq. $\left(10^{\prime \prime}\right)$ the internal flux $\beta$ becomes a single-valued function of $\beta_{x}$, if only the ac bias level becomes high enough to satisfy $\left|\gamma J_{0}(\alpha)\right| \leqslant 1$, and hence, the SQUID may enter the low-inductance regime even if $\gamma>1$.

In Sec. II B the relation between the ac bias current and the tank voltage will be derived. Before doing that we must find the ac component of the current circulating in the SQUID ring. From Eqs. (4)-(6) and (8) we have

$$
L_{R} I_{c}(t)=\Phi_{x}-M I \sin (\omega t-\theta)-\Phi_{\mathrm{dc}}-\Phi_{\mathrm{ac}} \sin \left(\omega t-\theta^{\prime}\right)
$$

and hence the ac part becomes, using Eqs. (9) and (11a),

$$
\begin{aligned}
I_{c}^{\mathrm{ac}}(t)= & -\left(M I / L_{R}\right) \sin (\omega t-\theta)+\left(M I / L_{R}\right) \\
& \times\left\{(1+\gamma \Gamma)^{2}+[\omega \tau(1+\epsilon \Gamma)]^{2}\right\}^{-1 / 2} \sin \left(\omega t-\theta^{\prime}\right) .
\end{aligned}
$$

If we further assume that $|\gamma \Gamma|<1$ and $|\epsilon \Gamma|<1$, the phase angle $\theta^{\prime}-\theta$ is in the first quadrant and

$\sin \left(\omega t-\theta^{\prime}\right)=\frac{(1+\gamma \Gamma) \sin (\omega t-\theta)-\omega \tau(1+\epsilon \Gamma) \cos (\omega t-\theta)}{\left\{(1+\gamma \Gamma)^{2}+[\omega \tau(1+\epsilon \Gamma)]^{2}\right\}^{1 / 2}}$.

Finally, introducing the short-hand notation

$$
1-\nu=\frac{1+\gamma \Gamma}{(1+\gamma \Gamma)^{2}+[\omega \tau(1+\epsilon \Gamma)]^{2}}
$$

and

$$
\mu=\frac{\omega \tau(1+\epsilon \Gamma)}{(1+\gamma \Gamma)^{2}+[\omega \tau(1+\epsilon \Gamma)]^{2}},
$$

the ac part of the circulating current is expressed as

$$
I_{c}^{\mathrm{ac}}(t)=-\left(M I / L_{R}\right) \nu \sin (\omega t-\theta)-\left(M I / L_{R}\right) \mu \cos (\omega t-\theta),
$$

which is valid in the low-inductance regime defined by the three inequalities

$$
\left|\gamma J_{0}(\alpha)\right| \leqslant 1, \quad\left|\gamma \frac{2 J_{1}(\alpha)}{\alpha}\right|<1, \quad \text { and } \quad\left|\epsilon \frac{2 J_{1}(\alpha)}{\alpha}\right|<1 \text { 。 }
$$

Most experiments ${ }^{916}$ indicate, in agreement with the theory, ${ }^{14}$ that the absolute value of the cosine-phi amplitude $\epsilon$ is less than unity such that the third inequality is always satisfied.

\section{B. The tank circuit}

The performance of the magnetometer circuit in Fig. 1 depends upon the amplitude modulation of the tank voltage $V(t)$ as the external de flux $\Phi_{x}$ is varied. The equations from which this voltage is determined are

$$
I_{p}(t)-I(t)=C \dot{V}(t)
$$

and

$$
V(t)=L \dot{I}(t)+r I(t)+M \dot{I}_{c}(t) .
$$

It will be shown below that the dominant contribution to the dc flux dependence of $V(t)$ arises from the dependence on flux of the current in the tank coil, $I(t)$. From Eqs. (16a) and (16b) this current is found to satisfy

$$
L C \ddot{I}(t)+M C \ddot{I}_{c}(t)+r C \dot{I}(t)+I(t)=I_{p}(t) .
$$

Inserting $I_{p}(t)=I_{p} \sin \omega t, I(t)$ from Eq. $(6)$, and $I_{c}(t)$ from Eq. (15) we find

$$
\begin{aligned}
& {\left[1-\omega^{2} L C\left(1-k^{2} \nu\right)\right] I \sin (\omega t-\theta)} \\
& +\omega r C\left[1+k^{2}(\omega L / r) \mu\right] I \cos (\omega t-\theta) \\
& =I_{p} \sin (\omega t),
\end{aligned}
$$

where the coupling coefficient $k^{2}=M^{2} / L L_{R}$ has been introduced.

Further introducing the resonant frequency, $\omega_{0}$ $=(L C)^{-1 / 2}$, and the quality factor, $Q_{0}=\omega_{0} r C=\omega_{0} L / r$, as well as the detuning factor, $\delta=Q_{0}\left(\omega_{0} / \omega-\omega / \omega_{0}\right)$, of the unloaded resonant circuit, we obtain

$$
\begin{aligned}
& \left(\delta+k^{2} Q_{0} \nu\right) I \sin (\omega t-\theta)+\left(1+k^{2} Q_{0} \mu\right) I \cos (\omega t-\theta) \\
& =Q_{0} I_{p} \sin (\omega t) .
\end{aligned}
$$

From Eq. (19) it follows that the tank circuit responds to flux changes both via modulation of the dispersion and of the dissipation in the tank circuit, since both the resonant frequency and the quality factor are flux dependent due to their dependence on the quantities $\nu$ and $\mu$, respectively. Generally, the two contributions to the flux dependence of $I$ cannot be separated. However, in the case, $\omega \tau=0$, which was considered by Hansma ${ }^{11}$ the mode is purely dispersive $(\mu=0)$, explaining why the SQUID is most sensitive to flux changes when biased at a frequency corresponding to the half-power points $(\delta= \pm 1)$ of the resonance.

Solving Eq. (19) for the current $I$ yields the result

$$
I=Q_{0} I_{p}\left[\left(\delta+k^{2} Q_{0} \nu\right)^{2}+\left(1+k^{2} Q_{0} \mu\right)^{2}\right]^{-1 / 2}
$$

and

$$
\theta=\arctan \left(\frac{1+k^{2} Q_{0} \mu}{\delta+k^{2} Q_{0} \nu}\right)
$$

Finally, the tank voltage, $V(t)=V_{T} \cos (\omega t-\psi)$, is found from Eq. (16b):

$$
\begin{aligned}
V_{T} \cos (\omega t-\psi)= & \omega L\left(1-k^{2} \nu\right) I \cos (\omega t-\theta) \\
& +r\left(1+k^{2} Q_{0} \mu\right) I \sin (\omega t-\theta) .
\end{aligned}
$$

The de flux dependence explicitly displayed here is less than that given implicitly through the dependence on $I$ by a factor of order $Q_{0}$. Also, the first term is larger than the second by the same order of magnitude. Hence, the voltage amplitude, $V_{T}$, is to a good approximation given by 


$$
V_{T} \approx \omega L I \text {. }
$$

Summarizing the results of the present derivation we have that the tank voltage is proportional to the current $I$ in the tank circuit inductance, which, in turn, is expressed in term of the internal ac flux $\alpha$ by

$$
I=\left(\Phi_{0} / 2 \pi M\right) \alpha\left\{(1+\gamma \Gamma)^{2}+[\omega \tau(1+\epsilon \Gamma)]^{2}\right\}^{1 / 2} .
$$

The internal ac flux is implicitly related to the ac bias current by Eq. (20a) and Eq. (11a):

$$
\begin{aligned}
\left(2 \pi M Q_{0} / \Phi_{0}\right) I_{p}= & \alpha\left\{(1+\gamma \Gamma)^{2}+[\omega \tau(1+\epsilon \Gamma)]^{2}\right\}^{1 / 2} \\
& \times\left[\left(\delta+k^{2} Q_{0} \nu\right)^{2}+\left(1+k^{2} Q_{0} \mu\right)^{2}\right]^{1 / 2},
\end{aligned}
$$

where

$$
\begin{aligned}
& \nu=\frac{\gamma \Gamma(1+\gamma \Gamma)+[\omega \tau(1+\epsilon \Gamma)]^{2}}{(1+\gamma \Gamma)^{2}+[\omega \tau(1+\epsilon \Gamma)]^{2}}, \\
& \mu=\frac{\omega \tau(1+\epsilon \Gamma)}{(1+\gamma \Gamma)^{2}+[\omega \tau(1+\epsilon \Gamma)]^{2}},
\end{aligned}
$$

and

$$
\Gamma=\frac{2 J_{1}(\alpha)}{\alpha} \cos \beta
$$

The internal dc flux $\beta$ is implicitly related to the external dc flux $\beta_{x}$ by

$$
\beta+\gamma J_{0}(\alpha) \sin \beta=\beta_{x} .
$$

In the limit $\gamma \ll 1$ and $\omega \tau=0$, the result stated above reproduces that a Hansma ${ }^{11}$ if the magnetic-flux-dependent screening terms in Eqs $s_{0}\left(10^{\prime}\right)$ and (11a) are neglected. The result to first order in $\gamma$ and $\omega \tau$ obtained by Rifkin et al. ${ }^{13}$ does not agree with that derived here. Neglecting flux-dependent screening effects as above and expanding to first order we get $\mu=\omega \tau[1+(\epsilon$ $-2 \gamma)$ ], whereas the corresponding relation from Ref. 13 can be shown to read $\mu=\omega \tau(1+\epsilon \Gamma)$. We will return to the significance of this deviation in Sec. IV.

The system of equations stated above does not allow the tank voltage $V_{T}$ to be expressed directly as a function of the external variables, $I_{p}$ and $\beta_{x}$. A numerical solution is however easily found proceeding as follows:

(1) The ac bias current $I_{p}$ and the externally applied dc flux $\beta_{x}$ is specified.

(2) A guess on $\alpha$ is made and $\beta$ is determined as the $n$th value, $\beta_{n}$, in the iteration $\beta_{n}=\beta_{x}-\gamma J_{0}(\alpha) \sin \beta_{n-1}$ 。 This trial solution is inserted into Eq. (23).

(3) Then, Eq. (23) is solved for $\alpha$ by repeated linear interpolations, continuously readjusting $\beta$ as described above for each new value of $\alpha$.

(4) Using the final pair of variables $(\alpha, \beta)$ the current $I$ and hence the tank voltage $V_{T}$ is determined from Eq. (11a) and Eq. (22), respectively. ${ }^{17}$

In order to illustrate the effects of the screening current, of a slight detuning, of the $G L_{R}$ time constant, and of the cosine-phi amplitude we have evaluated the tank voltage as described above, and the results are presented in Sec. III.

\section{NUMERICAL RESULTS}

Before going into details with the calculations it is instructive to consider a particularly simple limiting case. From Eq. (11c) it follows that $\Gamma$ approaches zero in an oscillating fashion as $\alpha$ is increased. Using index " $\infty$ " for the resulting linear asymptotic relationship, we have

$$
\begin{aligned}
\left(2 \pi M Q_{0} / \Phi_{0}\right) I_{p}= & \alpha_{\infty}\left[1+(\omega \tau)^{2}\right]^{1 / 2}\left[\left(\delta+k^{2} Q_{0} \nu_{\infty}\right)^{2}\right. \\
& \left.+\left(1+k^{2} Q_{0} \mu_{\infty}\right)^{2}\right]^{1 / 2}
\end{aligned}
$$

and

$$
V_{T_{\infty}}=\omega L Q_{0} I_{p}\left[\left(\delta+k^{2} Q_{0} \nu_{\infty}\right)^{2}+\left(1+k^{2} Q_{0} \mu_{\infty}\right)^{2}\right]^{-1 / 2},
$$

where

$$
\nu_{\infty}=(\omega \tau)^{2} /\left[1+(\omega \tau)^{2}\right]
$$

and

$$
\mu_{\infty}=\omega \tau /\left[1+(\omega \tau)^{2}\right] .
$$

In the linear limit the conductance $G$ carries the total current circulating in the SQUID ring and the only effect of the SQUID on the tank circuit is to shift the resonant frequency from $\delta=0$ to

$$
\delta_{\mathrm{res}}=-k^{2} Q_{0}(\omega \tau)^{2} /\left[1+(\omega \tau)^{2}\right] .
$$

From the asymptotic slope at resonance of the $V_{T}$-vs$I_{p}$ characteristic it follows that the quality factor is changed from $Q_{0}$ to $Q_{\text {eff }}$, where

$$
Q_{\text {eff }}^{-1}=Q_{0}^{-1}+k^{2} \omega \tau /\left[1+(\omega \tau)^{2}\right] .
$$

The quality factor, $Q_{0}$, and the resonant frequency, $\omega_{0} / 2 \pi$, of the unloaded tank circuit may be measured directly in the presence of a SQUID ring with the weak link open-circuited $(\omega \tau=0)$. The coupling coefficient, $k^{2}$, as well as $\omega \tau$ may then be determined using Eq. (27)

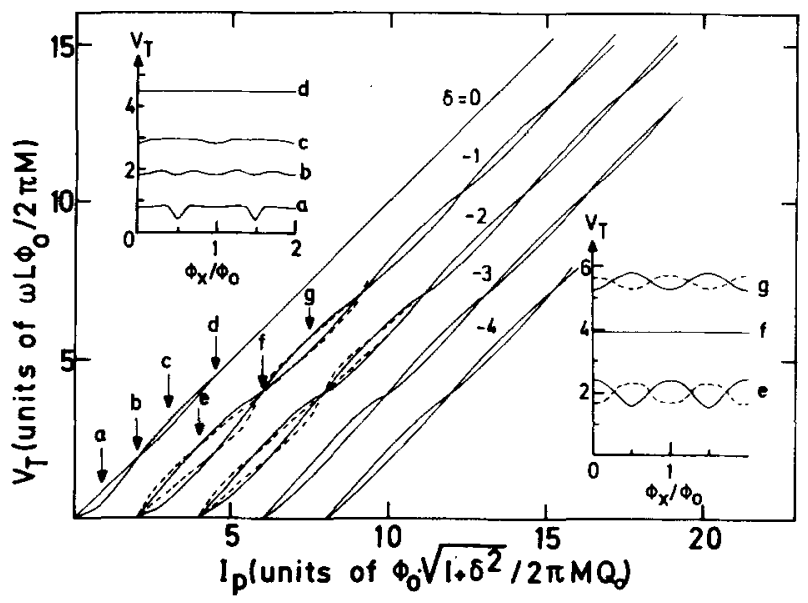

FIG. 2. ac voltage-current characteristics calculated for $\omega \tau$ $=0$ with the detuning factor $\delta$ and the external de flux $\Phi_{x}$ as parameters. The solid lines are calculated for $\delta=0$ to -4 as indicated, whereas the dashed lines are for $\delta=1$ and $\delta=2$, respectively. For each $\delta$ traces are shown for $\Phi_{x}=n \Phi_{0}$ and $\Phi_{x}$ $=\left(n+\frac{1}{2}\right) \Phi_{0}$. The traces for different $\delta$ are displaced horizontally for the sake of clarity. The insets show the tank voltage amplitude $V_{T}$ in units of $\omega L \Phi_{0} / 2 \pi M$ as a function of $\Phi_{x} / \Phi_{0}$ at values of ac bias current as indicated by the arrows. The circuit parameters are $\gamma=0.75, Q_{0}=100$, and $k^{2} Q_{0}=1$. 


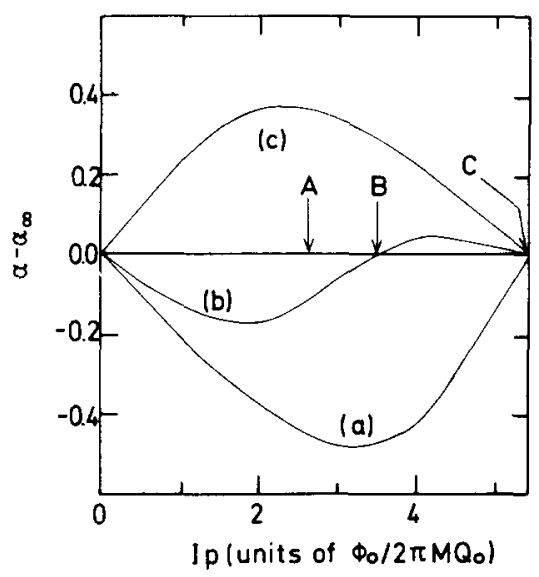

FIG. 3. The difference between the internal and external normalized ac flux as a function of ac bias current with circuit parameters $\gamma=0.75, Q_{0}=100$, and $k^{2} Q_{0}=1$. We have used $\omega \tau$ $=0$ and $\delta=-1$, implying that $I_{p}=\left(\Phi_{0} \sqrt{2} / 2 \pi M Q_{0}\right) \alpha_{\infty}$. and (1) $\Phi_{x}$ $=n \Phi_{0}$ : (b) $\Phi_{x}=\left(n+\frac{1}{4}\right) \Phi_{0}$ or $\left(n+\frac{3}{4}\right) \Phi_{0}$, (c) $\Phi_{x}=\left(n+\frac{1}{2}\right) \Phi_{0}$.

and Eq. (28) from the asymptotic behavior at resonance of the reestablished ring.

Returning briefly to the final equations of Sec. II we find that for $\Phi_{x}=n \Phi_{0}$ and $\Phi_{x}=\left(n+\frac{1}{2}\right) \Phi_{0}\left[\beta_{x}=0\right.$ and $\beta_{x}=\pi$; $\bmod (2 \pi)]$ the equations are satisfied consistently with $\alpha=\alpha_{\infty}$, provided that the ac bias current, $I_{p}$, is adjusted such that $J_{1}\left(\alpha_{\infty}\right)=0$ with $\alpha_{\infty}$ from Eq. (24). In other words, the distance on the current axis between nodes in the $V_{T}$-vs $-I_{p}$ characteristic is given by the distance between zeros of the first-order Bessel function and is, via Eq. (24), related to the mutual inductance, $M{ }^{18}$

Below, the numerical evaluations of the equations derived in Sec. II are presented. First, the simple case is considered, where the junction conductance is neglected $(G=0)$. Figure 2 shows the tank voltage as a function of ac bias current for $\gamma=0.75$, which is sufficiently large for the screening current to be important. We have used $k^{2} Q_{0}=1$ in the calculation. ${ }^{19}$ The solid lines in Fig. 2 show the ac voltage-current characteristic for $\delta=0,-1, \ldots,-4$, and with the external dc flux $\Phi_{x}=n \Phi_{0}$ and $\Phi_{x}=\left(n+\frac{1}{2}\right) \Phi_{0}$, respectively. The dashed lines show the low-current parts of the voltage-current characteristics for $\delta=+1$ and +2 . The results shown in Fig. 2 should be compared to the result of the firstorder calculation by Hansma (Fig. 3 of Ref. 11). When the large value of $\gamma$ and the effects of the screening current are properly taken into account, we find, first, that a magnetic flux dependence is present even at resonance $(\delta=0)$; second, that the lobe pattern has been distorted; and third, that the characteristics for positive and negative $\delta$ are different although their asymptotic slopes are identical. The insets of Fig. 2 show the magnetic flux modulation of the tank voltage for values of the ac bias current indicated by the arrows. We note a strong distortion of the simple sinusoidal dependence expected from a first-order calculation. The apparent node in the lobe pattern at arrow (b), for instance, does not reflect that the tank voltage at that particular bias point is independent of the mag-

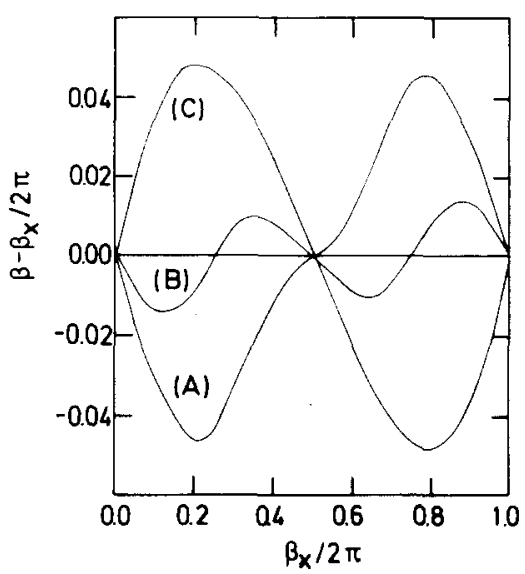

FIG. 4. The difference between the internal and external normalized dc flux as a function of normalized external dc flux for $\gamma=0.75, Q_{0}=100, k^{2} Q_{0}=1, \omega \tau=0$, and $\delta=-1$. The traces (A), (B), and (C) a re calculated for values of the ac bias current indicated by the corresponding arrows in Fig. 3.

netic flux, but rather that the period of oscillation is $\frac{1}{2} \Phi_{0}$ as evident from trace (b) of the inset.

In order to illustrate further the effect of screening, we have in plotted Fig. 3 the difference between the internal ac flux $\alpha$ and the asymptotic value $\alpha_{\infty}$ (which also may be interpreted as the externally applied ac flux), as a function of $I_{b}$, for $\delta=-1$ and for the external dc flux $\beta_{x}$ equal to $0, \frac{1}{2} \pi$ and $\frac{3}{2} \pi$, and $\pi[\bmod (2 \pi)]$. This is shown in traces (a), (b), and (c), respectively.

Whereas Fig. 3 demonstrates the effect of screening in the ac regime, the effect of screening at dc is illustrated in Fig. 4, where the difference between the internal and the external dc flux is plotted as a function of the external dc flux for $\delta=-1$. The traces (A), (B), and (C) are calculated for values of the ac bias current indicated by the corresponding arrows in Fig. 3. The ac

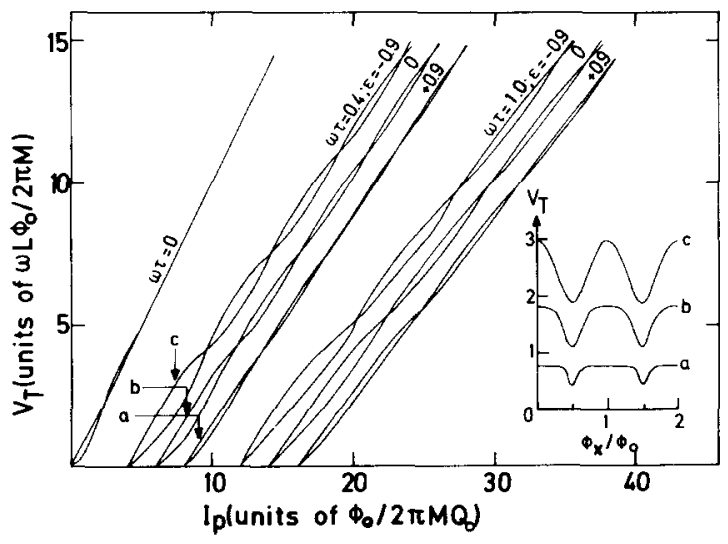

FIG. 5. ac voltage-current characteristics calculated for $\delta=0$ with $\omega \tau=0,0.4$, and 1.0 , with $\epsilon=0.9,0.0$, and +0.9 , and with $\Phi_{x}=n \Phi_{0}$ and $\left(n+\frac{1}{2}\right) \Phi_{0}$. The traces for different $\omega \tau$ and $\epsilon$ are displaced horizontally for the sake of clarity. The trace $\omega \tau=0$ which is independent of $\epsilon$ is also shown in Fig. 2. The inset shows the tank voltage as a function of $\Phi_{x} / \Phi_{0}$ for $\omega \tau=0.4$ and $\epsilon=+0.9,0.0$, and -0.9 , traces (a), (b), and (c), respectively. The ac bias current is adjusted to the maximum of the first lobe as shown by the corresponding arrows. 

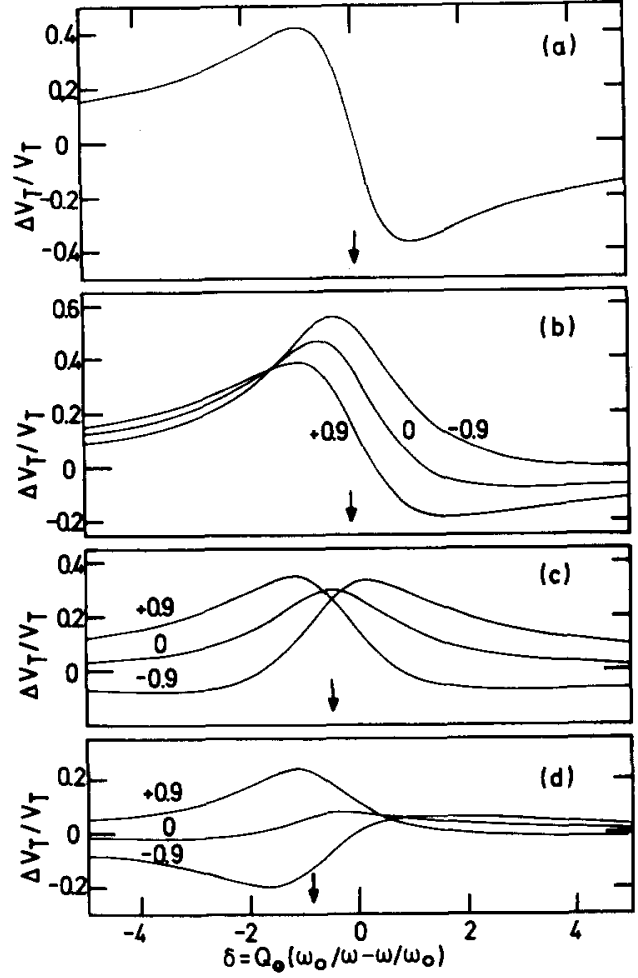

FIG. 6. Relative modulation depth, $\Delta V_{T} / V_{T}$, as a function of detuning factor with $\epsilon$ as a parameter. All traces are calculated using $\gamma=0.75, Q_{0}=100, k^{2} Q_{0}=1$, and with the ac bias current adjusted such that $J_{1}\left(\alpha_{\infty}\right)$ is at its first maximum. (a) $\omega \tau=0$; (b) $\omega \tau=0.4$; (c) $\omega \tau=1.0$; (d) $\omega \tau=2.6$. The arrows indicate the detuning factor, $\delta_{\text {res }}$, at resonance.

bias has been selected such that: (A) $J_{1}\left(\alpha_{\infty}\right)$ is at its first maximum, (B) $J_{0}\left(\alpha_{\infty}\right)$ has reached its first zero, and $(C) J_{1}\left(\alpha_{\infty}\right)$ has reached its second zero.

From Figs. 2-4 it follows that when the parameter $\gamma$ approaches the upper limit for the low-inductance regime it is important to take the effect of screening into account.

The screening is equally important when $\omega \tau$ is nonzero, but then also the cosine-phi term affects the behavior. This is shown in Fig. 5, where some selected ac voltage-current characteristics are plotted. All traces are calculated with $\delta=0$, with $k^{2} Q_{0}=1$, and with $\gamma=0.75$. The values of $\omega \tau$ and of the cosine-phi amplitude $\epsilon$ are as shown in Fig. 5. Qualitatively, these ac voltage-current characteristics are very similar to those shown in Fig. 2. The effect of a nonzero $\omega \tau$ is mainly to change the asymptotic slope and to stretch the lobes to span a larger interval of ac bias current. The cosine-phi amplitude affects the amplitude modulation of the tank voltage as shown in the inset. Here, the dc flux dependence is shown for fixed $\omega \tau$, biased at the current of maximum modulation depth, and with $\epsilon$ as a parameter. Procedures, which might allow the magnitude of $\epsilon$ to be extracted from experimental data, will be discussed in more detail in Sec. IV. In the remaining part of this section the consequence of the existence of an upper limit on the bias frequency will be further illucidated.

Whether the ac SQUID may be utilized as a sensitive magnetometer depends on the magnitude of the relative modulation depth, $\Delta V_{T} / V_{T}$, which here is defined as the ratio of $\Delta V_{T}=V_{T}\left(\beta_{x}=0\right)-V_{T}\left(\beta_{x}=\pi\right)$ to $V_{T}\left(\beta_{x}=\frac{3}{2} \pi\right)$. We have evaluated $\Delta V_{T} / V_{T}$ as a function of the detuning factor $\delta$ with $\omega \tau$ as a parameter, and at a bias current such that $J_{1}\left(\alpha_{\infty}\right)$ is at its first maximum. According to Eq. (24) this implies that in order to keep $\alpha_{\infty}$ constant, $I_{p}$ must be constantly readjusted as $\delta$ and $\omega \tau$ are varied. As above, we have used $\gamma=0.75$ and $k^{2} Q_{0}=1$. The cosine-phi amplitude does play an important role, and accordingly, three values of $\epsilon$ have been assumed: $\epsilon=0.9, \epsilon=0$, and $\epsilon=-0.9$. Some representative results are shown in Fig. 6. In Fig. 6(a) we have $\omega \tau=0$, and the response is almost an odd function of $\delta$. As $\omega \tau$ increases, the resonant frequency shifts in agreement with $\mathrm{Eq}$. (27) indicated by the arrow in the figure. Figures $6(\mathrm{~b}), 6(\mathrm{c})$, and $6(\mathrm{~d})$ are calculated for $\omega \tau=0.4,1.0$, and 2.6 , respectively. For increasing $\omega \tau$ the positive peak dominates at first and mostly so for negative $\epsilon$. For larger $\omega \tau$ the positive peak disappears, slowly for $\epsilon>0$ and more rapidly for $\epsilon \leqslant 0$. For $\epsilon<0$, however, a negative peak appears which soon dominates the response as it moves towards the resonant condition, $\delta=-1$, when $\omega \tau \rightarrow \infty$. The response extrema are plotted in Fig. 7 (the solid lines) as a function of $\omega \tau$ and with $\epsilon$ as a parameter. This plot illustrates the important results that, provided the cosinephi term is nonzero, an appreciable magnetic dc flux dependence persists at high pump frequencies, that the behavior in the high-frequency limit is actually dominated by the phase-dependent conductivity, and hence, that the response is very sensitive to the magnitude of that term.

To this point we have used $k^{2} Q_{0}=1$ in the evaluation in order to keep the number of variables to a minimum. From Eq. (28) it follows that the losses in the circuit are in fact frequency dependent if $\omega \tau$ is nonzero. Hence, in order to compensate for the dissipation in the junction conductance, the coupling should rather be adjusted such that $k^{2} Q_{e f f}=1$ is satisfied. The response maxima

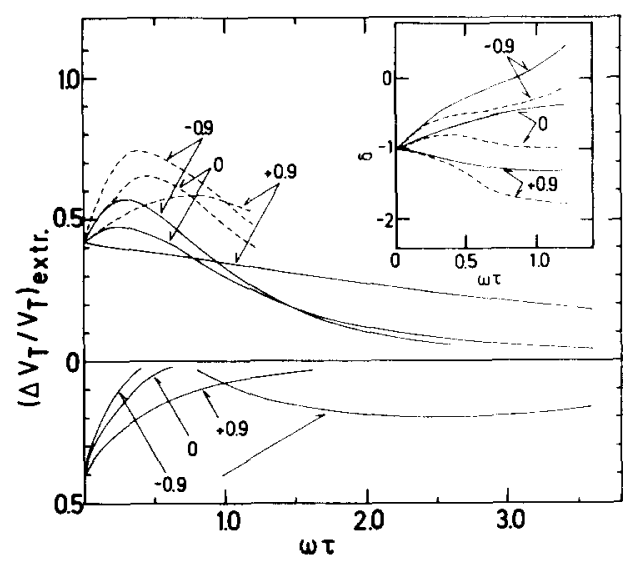

FIG. 7. Extremal values of the relative modulation depth as a function of $\omega \tau$ with $\epsilon$ as a parameter. The coupling coefficient is adjusted such that $k^{2} Q_{0}=1$ (solid lines) or such that $k^{2} Q_{\text {eff }}$ $=1$ (dashed lines). Other circuit parameters are $\gamma=0.7 \overline{5}$ and $Q_{0}=100$. The inset shows the position in the $\omega \tau-\delta$ plane of the point of maximum relative modulation depth. 


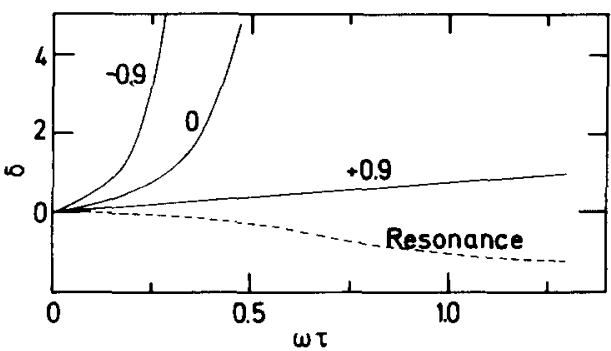

FIG. 8. Contours of zero modulation depth as a function of $\omega \tau$ with $\epsilon$ as a parameter (solid lines). The detuning factor at resonance $\delta_{\text {res }}$ is also shown as a function of $\omega \tau$ (dashed line).

Here the ac bias current is adjusted such that $\alpha_{\infty}=2.0$ at resonance. The coupling coefficient is adjusted to maintain $k^{2} Q_{\text {eff }}$ $=1$ as $\omega T$ is varied.

have been recalculated using the modified coupling coefficient, and these results are also shown in Fig. 7 (the dashed lines).

We note that the maximal relative modulation depth may peak at a finite value of $\omega \tau$. When the coupling coefficient is adjusted such that $k^{2} Q_{\text {eff }}=1$ the phenomenon is strongly enhanced compared to the case, $k^{2} Q_{0}=1$, and it occurs for all values of $\epsilon$. Furthermore, the peak in the maximal response has been pushed to a higher value of $\omega \tau$. Since $Q_{\theta f s}$ approaches $Q_{0}$ as $\omega \tau$ is increased, the high-frequency behavior remains unchanged. The enhancement of the response compared to the $\omega \tau=0$ case may be as large as $40-80 \%$ depending on $\epsilon$.

\section{DISCUSSION OF THE COSINE.PHI DEPENDENCE}

All attempts to extract the magnitude and sign of the cosine-phi amplitude from experimental data ${ }^{16}$ have produced results with the magnitude consistent with the tunneling theory ${ }^{14}$ but with the opposite sign. We have demonstrated in Sec. III that the ac-SQUID response is a strong function of the cosine-phi amplitude. We did, however, emphasize the effect of a variation of $\omega \tau$, which in a real experiment is a rather impractical approach. ${ }^{20}$

If $\omega \tau$ is fixed it should be possible to determine $\epsilon$ using a curve-fitting procedure to obtain agreement between, for instance, an experimental-response-vsdetuning curve and the corresponding theoretical plot. As is evident from Fig. 6 such an approach will be particularly valuable for large values of $\omega \tau$ where the cosine-phi term dominates the response. Alternatively, it might be simpler to select a specific feature such as the position in the $\omega \tau-\delta$ plane of the point of maximum response, or, as suggested by Hansma, ${ }^{12}$ the position of the point of zero response. However, neither the former nor the latter of these suggestions allows $\epsilon$ to be determined directly. As shown in the inset in Fig. 7 the contours of maximum response does depend strongly on $\epsilon$ but also on how the coupling is adjusted. That the contours of zero response is also not simply related to the magnitude of $\epsilon$ is demonstrated in Figs. 8 and 9 . As above, we have used $\gamma=0.75$ and $k^{2} Q_{e t 1}=1$ but the ac current, $I_{p}$, is now adjusted such that the external ac flux, $\alpha_{\infty}$, attains a specified value at resonance $\left(\delta_{\text {res }}=-k Q_{0} \nu_{\infty}\right)$ and $I_{p}$ rather than $\alpha_{\infty}$ is kept constant as $\delta$ is varied away from resonance.

In Figs. 8 and $9(\mathrm{~b})$ the ac bias current has been adjusted such that the point of zero response occurs at $\delta=0$ for $\omega \tau=0$, i. e. , the bias point is at arrow (b) in Fig. 2. In Figs. 9 (a) and 9 (c) the tank circuit is biased at a slightly smaller and a slightly larger current, respectively.

The first-order calculation of Hansma $\mathrm{ma}^{12}$ and of Rifkin et $a l .{ }^{13}$ led to the conclusion that the point of zero response occurred above, at, or below resonance depending on $\epsilon$ being positive, zero, or negative. The present analysis shows that in the first-order limit without screening the point of zero response occurs at $\delta_{0}=(2-\epsilon / \gamma) \omega \tau$. Hence, the crucial parameter is $2 \gamma-\epsilon$ rather than $\epsilon\left(\delta_{\text {res }}=0\right.$ to first order $)$. In general the result is more complicated. As shown in Fig. 9 the point of zero response may occur above or below resonance depending on $\omega \tau$ and on the bias current as well as on $\epsilon$ and $\gamma$.

We hence conclude that although the detailed behavior of the ac SQUID is strongly dependent on the phasedependent conductivity, the value of all other circuit parameters must be accurately known in order to extract the magnitude of the cosine-phi amplitude. The parameters $\omega \tau$ and $k^{2} Q_{0}$ may be determined directly from the asymptotic behavior of the ac voltage-current characteristic at resonance as discussed in Sec. III. Thus, only the $L_{R} I_{0}$ product $(\gamma)$ and the cosine-phi amplitude $(\epsilon)$ remain as unknown parameters, which in turn must be determined by means of a two-parameter fit to the experimental results.

\section{SUMMARY AND CONCLUSION}

The magnetometer circuit consisting of a resonant circuit magnetically coupled to a superconducting ring containg a single Josephson junction was analyzed. The analysis assumed a single-valued relation between the

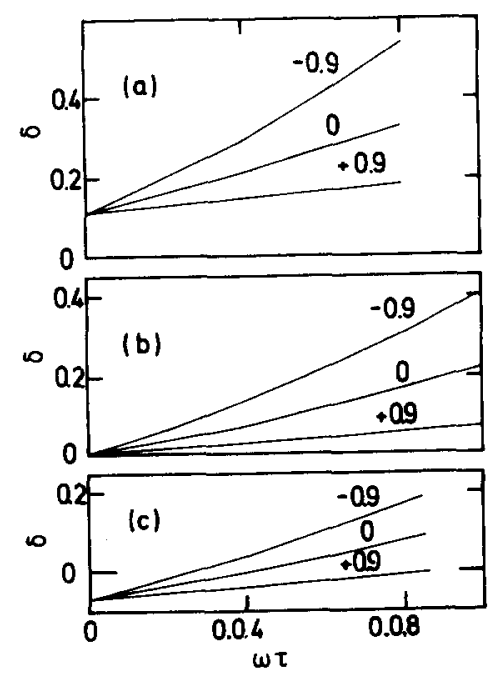

FIG. 9. Contours of zero modulation depth as a function of $\omega \tau$ and with $\epsilon$ as a parameter. The circuit parameters are adjusted as in Fig. 8. However, different values of $\alpha_{\infty}$ at resonance are used. (a) $\alpha_{\infty}=1.5$; (b) $\alpha_{\infty}=2.0$; (c) $\alpha_{\infty}=2.6$. 
magnetic flux enclosed in the ring and the externally applied flux. This assumption was satisfied in the lowinductance regime defined by the two inequalities $\left|\gamma J_{0}(\alpha)\right| \leqslant 1$ and $\left|2 \gamma J_{1}(\alpha) / \alpha\right|<1$, where $\gamma$ was the normalized maximum screening flux and $\alpha$ the normalized enclosed ac flux. The Josephson junction was described by the resistively shunted junction model including a phase-dependent term in the shunt conductance. Within this model the circuit equations was solved. The solution was valid for arbitrary pump frequency and the influence of the screening current circulating in the SQUID ring was taken into account. Only one additional assumption was made which might restrict the general validity of the result, namely, that only the dc component and the component at the pump frequency of the induced currents are important, the argument being that the resonant circuit does not respond to the higher harmonics. This assumption is reasonable provided that (a) the pump frequency is close to the resonant frequency and that (b) the quality factor of the resonator is large $\left(Q_{0} \geqslant 100\right)$.

The analysis led to relations expressing explicitly the tank voltage, the ac bias current, and the magnetic dc flux in terms of the internal variables, i. e., the ac and dc flux enclosed in the SQUID ring. These relations were easily evaluated numerically.

Several important properties of the magnetometer circuit was illustrated. First, we found that the screening current must be taken into account in order to understand the detailed behavior of the SQUID. Second, that the cosine-phi-dependent conductivity became increasingly important and eventually dominated the SQUID response as the product, $\omega \tau$, of the pump frequency $\omega$ and the time constant $\tau$ of the SQUID ring was made larger. Third, that in general both the dispersion and the dissipation in the tank circuit depended on the dc flux applied to the SQUW ring, and that the two effects could cooperate as well as counteract each other in producing the amplitude modulation of the tank voltage. As a consequence the SQUID response peaked at a finite value of $\omega \tau$ if appropriately biased. Finally, the effect of the cosine-phi amplitude was discussed in more detail. The properties of the system was found strongly dependent on $\epsilon$ particularly for large $\omega \tau$. Nevertheless, no straightforward method was found by means of which the magnitude of $\epsilon$ could be measured. Whereas all parameters, except the cosine-phi amplitude and the maximum screening flux, could be determined from the asymptotic behavior for large bias currents, the remaining parameters must be determined from a two-parameter fit to the experimental results.

In conclusion we can state that it was possible to derive a general expression describing the properties of a simple magnetometer circuit in the low-inductance regime. The derivation was based on a phenomenological junction model which has proven adequate in many other contexts. One important aspect of the problem has however been left open. That is, the effect of the junction generated noise on the limiting sensitivity of the system considered as a low-frequency magnetometer.

\section{ACKNOWLEDGMENT}

N. F。 Pedersens critical reading of the manuscript is gratefully acknowledged.

${ }^{1}$ A. H. Silver and J.E. Zimmerman, Phys. Rev. 157, 317 (1967).

2. J. G. Goodkind and D. L. Stolfa, Rev. Sci. Instrum. 41, 799 (1970).

${ }^{3}$ J. E. Zimmerman, J. Appl. Phys. 42, 30 (1971).

${ }^{4}$ R. P. Giffard, R. A. Webb, and J.C. Wheatley, J. Low Temp Temp. Phys. 6, 533 (1972).

${ }^{5}$ W. W. Webb, IEEE Trans. Magn. MAG-8, 51 (1972).

${ }^{6} \mathrm{~J}$. Clarke, Proc. IEEE 61, 8 (1 973).

${ }^{7}$ W. L. Goodman, V.W. Hesterman, L. H. Rorden, and W. S. Goree, Proc. IEEE 61, 20 (1973).

${ }^{8}$ J.E. Zimmerman, P. Thiene, and J.T. Harding, J. Appl. Phys. 41, 1572 (1970).

${ }^{9}$ D. A. Vincent and B. S. Deaver, Jr., Phys. Rev. Lett. 32 , 212 (1974).

${ }^{10} \mathrm{~J}$. E. Zimmerman, J.Appl. Phys. 42, 4483 (1971).

${ }^{11}$ P. K. Hansma, J. Appl. Phys. 44, 1491 (1973).

${ }^{12}$ P. K. Hansma, Bull. Am. Phys. Soc. 18, 1609 (1973).

${ }^{13}$ R. Rifkin, D. A. Vincent, P.K. Hansma, and B.S. Deaver, Jr., IEEE Trans. Magn. MAG-11, 873 (1975).

${ }^{14}$ B. D. Josephson, Adv. Phys. 14, 419 (1965); U.K. Poulsen, Rev. Phys. Appl. 9, 41 (1974); R. E. Harris, Phys. Rev. B 10, 84 (1 974).

${ }^{15}$ W. C. Stewart, Appl. Phys, Lett. 12, 277 (1968); D. E. McCumber, J. Appl. Phys. 39, 3113 (1968).

${ }^{16}$ N.F. Pedersen, T.F. Finnegan, and D. N. Langenberg, Phys. Rev. B 6, 4151 (1972); C. M. Falco, W.H. Parker, and S. E. Trullinger, Phys. Rev. Lett. 31, 933 (1973); 31, 1476 (1973); S. Wolf and M. Nisenoff, Bull. Am. Phys. Soc. 19,205 (1974).

${ }^{17} \mathrm{~A}$ solution by iteration was attempted. With initial values $\Gamma_{1}=0$ and $\beta_{1}=\beta_{x}, \alpha_{1}$ was calculated from Eq. (23). Then a value $\Gamma_{2}$ was calculated from Eq. (11c) and $I$ was calculated from Eq. (20a). The next step was a recalculation of $\beta=\beta_{2}$ where $\beta_{2}=\beta_{x}-\gamma J_{0}\left(\alpha_{1}\right) \sin \beta_{1}$ and of $\alpha_{2}$ from Eq. (11a) which in turn permitted a calculation of $\Gamma_{3}$ from Eq. (11 c). The procedure was repeated and was found to converge rapidly in most cases. For some choices of parameters, however, the scheme did not converge and was hence abandoned in favor of the safer but also somewhat slower interpolation scheme described in the text.

${ }^{18} \mathrm{An}$ independent calibration of the mutual inductance is possible based on the periodicity of the $V_{T}$-vs- $\Phi_{x}$ trace, provided that the tank inductance is simultaneously used as a dc coil.

${ }^{19}$ The optimum coupling condition $k^{2} Q_{0}=1$ was found empirically by Simmonds and Parker [M. B. Simmonds and W. H. Parker, J. Appl. Phys. 42, 38 (1971)]. It has been argued that this condition follows from the requirement that the maximum energy stored in the SQUD ring is dissipated in the tank circuit in each cycle (D. B. Sullivan, NBS Technical Note No. 629, 1972 (unpublished) p. 17l. From the argument above one actually obtains $k^{2} Q_{0}=2 \pi$ in qualitative agreement with the results of Jackel et al. [(L.D. Jackel, T.D. Clark, and R.A. Buhrman, IEEE Trans. Magn. MAG-11, 732 (1972)]. However, whether one or the other of these values is used does not introduce any fundamental modifications of the SQUD behavior in the context of this paper.

${ }^{20} \omega \tau$ may be varied using a point-contact SQUID with adjustable contact pressure. Then also the temperature should be varied in order to keep the critical current (or $\gamma$ ) constant. This is permitted only if $\epsilon$ is independent of the temperature and according to tunneling theory this is the case only in the zero voltage limit of $\epsilon$. 